\title{
Teenager with an irritable hip, anaemia and malaise
}

\author{
R Hertzberger-ten Cate, B A C Dijkmans, F C Breedveld
}

\author{
Department of \\ Paediatrics, \\ University Hospital \\ Sophia Children's \\ Hospital, \\ Rotterdam, \\ The Netherlands \\ R Hertzberger-ten Cate \\ Department of \\ Rheumatology, \\ University Hospital \\ Leiden, \\ The Netherlands \\ B A C Dijkmans \\ F C Breedveld \\ Correspondence to: \\ Dr B A C Dijkmans, \\ University Hospital, \\ Department of \\ Rheumatology, \\ Building 1, C4-R \\ P O Box 9600, \\ 2300 RC Leiden, \\ The Netherlands.
}

\section{Case report}

An 11 year old Moroccan boy was referred to an orthopaedic surgeon in another hospital with a two year history of pain in the left groin, and limping. He was the third child of healthy unrelated parents who moved to the Netherlands in 1970 . The pregnancy and delivery were uncomplicated. The boy was breast fed for six months. His vaccinations were according to the Dutch scheme with, in addition, BCG vaccination. He had been healthy throughout his youth, with normal physical and mental development. An older brother had arthralgias of a shoulder and knee, with prolonged morning stiffness without manifest swelling of the joints. At one time this brother had suffered an acute painful redness of one eye. Family history disclosed no other rheumatic diseases.

Height $(\mathrm{cm})$

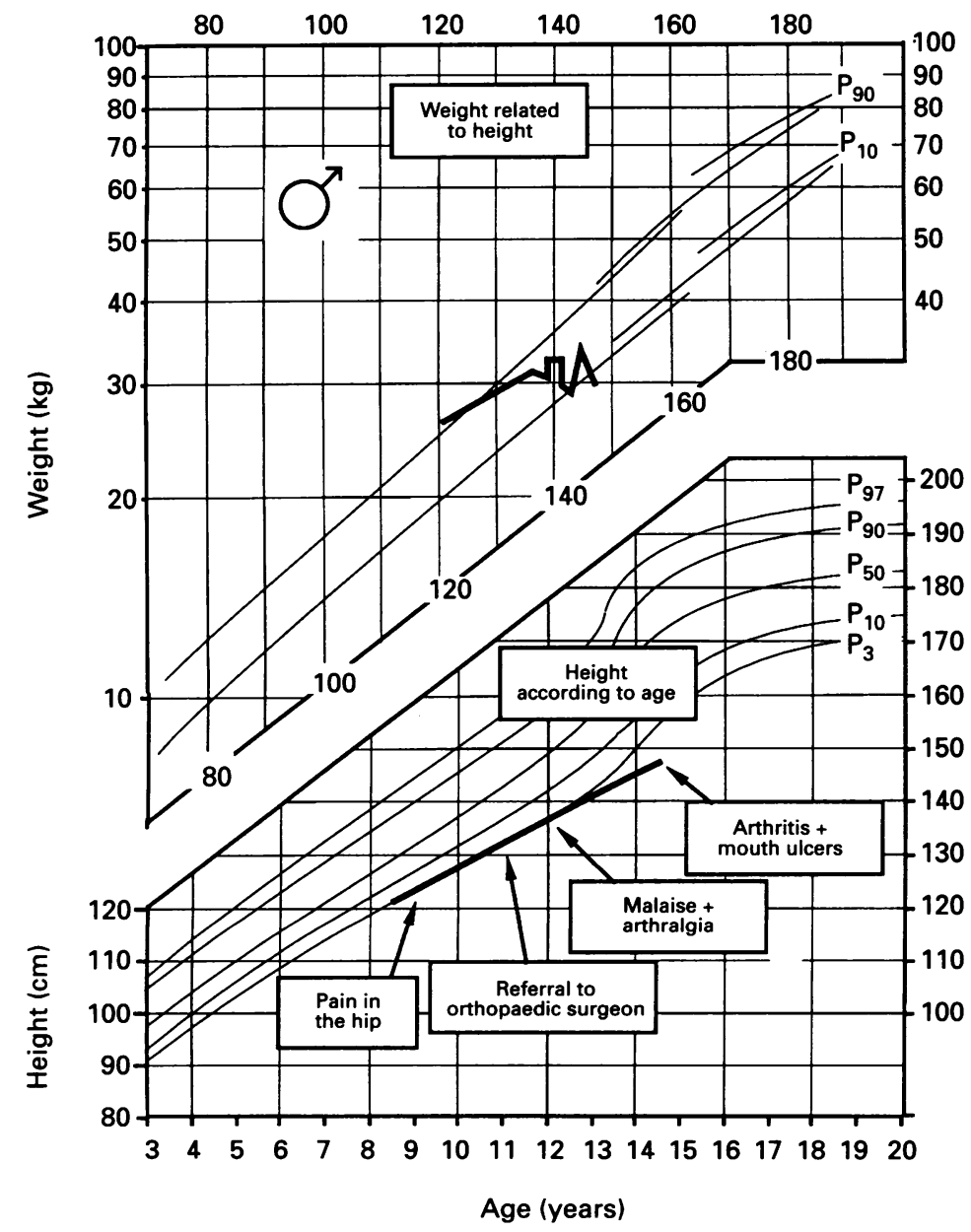

Figure 1 Growth curve of a teenager with an irritable hip, anaemia and malaise.
The joint problems in our patient started without previous infection or trauma. $\mathrm{He}$ intermittently complained of pain in the left groin with limping and morning stiffness, especially the day after playing soccer. After two years the general practitioner referred the boy to an orthopaedic surgeon who could not find any abnormality on physical examination. A full blood test showed a low haemoglobin $(7 \cdot 0 \mathrm{mmol} / \mathrm{l})$ with a packed cell volume (PCV) of 0.33 and a mean corpuscular volume (MCV) of 78. A radiograph of the pelvis revealed no abnormalities. The joint complaints were diagnosed as 'irritable hip' and the anaemia as the result of insufficient iron intake. Follow up was by the general practitioner, who prescribed oral iron and physiotherapy. Subsequently, after each exercise session the hip pain increased for several days with prolongation of morning stiffness, improving with a hot shower and walking. One year later the general practitioner was consulted by the youth because of general malaise, anorexia, and pain in the left groin and both knees. Blood tests yielded haemoglobin $6.7 \mathrm{mmol} / \mathrm{l}$, PCV 0.31 , a normal leucocyte count and an increased erythrocyte sedimentation rate (ESR) (Kowarski's method) of $38 \mathrm{~mm} / 1 \mathrm{st} \mathrm{h}$ (normal value less than $10 \mathrm{~mm}$ ) $1 \mathrm{st} h$ ). The boy felt that his growth was insufficient. He was referred to a paediatrician for evaluation of anaemia. Additional questions about possible extra-articular manifestations revealed an altered defaecation pattern with two or three loose stools per day. Further questioning about the presence of fever, skin symptoms, or acute red eyes elicited negative responses. On physical examination the boy was pale, without looking ill. There were no skin, nail, or mucosal abnormalities. His weight was on the 50th percentile and his height on the 3 rd percentile (fig 1). There were several small lymph nodes palpable in both axillae and groins. The spleen extended just below the costal margin. No joints were swollen, tender, or warm on palpation. There were no limitations in movement on physical examination; however, flexion, rotation, and extension of the left hip joint were painful.

Laboratory investigation confirmed hypochromic normocytic anaemia, otherwise the full blood count and film were normal. Liver and renal function tests were normal. Immunoglobulin levels were not increased. Serum antibodies to toxoplasma, mycoplasma, cytomegalovirus, Epstein-Barr virus, listeria and Borrelia burgdorferi were absent. Culture of throat swab was negative. Cultures of stools were negative for salmonella, shigella, Yersinia 


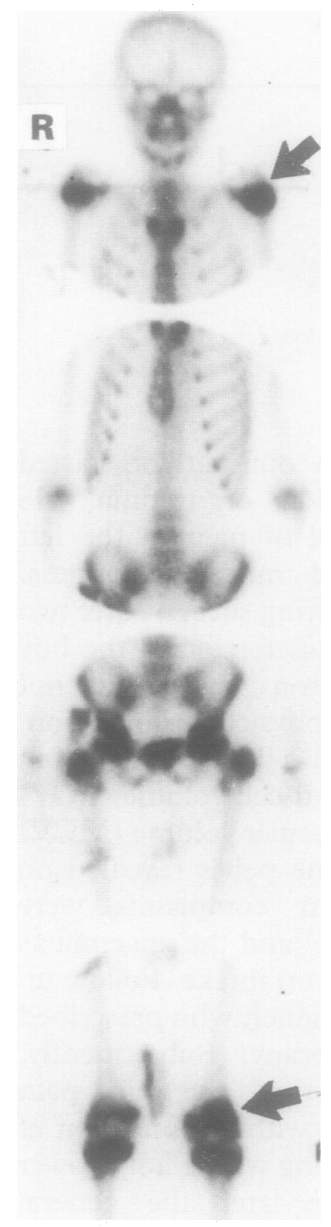

Figure 2 Technetium scan. The arrows indicate and the left knee. enterocolitica and campylobacter; Entamoeba coli and Endolimax nana cysts were seen, but were not considered to be pathogenic. Faecal occult blood tests were repeatedly negative. IgA antigliadine and antireticulin antibodies were absent. Circulating IgM rheumatoid factor, antinuclear antibodies, or antibodies to extractable nuclear antigens could not be detected. A chest radiograph, abdominal ultrasound, and radiographs of knees and pelvis showed no abnormalities. The $\mathrm{H} 2$ breathing test (as screening for lactose malabsorption) was strongly abnormal. Because of the suspicion of lactase deficiency, the patient was treated with supplementary lactase and a diet. These measures gave some improvement in the general wellbeing, and an increase in weight.

After three months, the patient again complained of weight loss and arthralgia of the left ankle and left shoulder. Both hips were now limited in movement. The paediatric rheumatologist suggested determination of the HLA-B27 antigen, which proved to be present. Occult blood tests in the stools were now positive. A technetium scan revealed hot spots in the left shoulder and the left knee (fig 2).

The patient was seen by the paediatric rheumatologist several weeks later. At that time there was marked malaise, painful sores in the mouth and residua of a red papulous skin disorder under the sole of the foot, resembling keratodermia blennorrhagica. Pain was indicated by the patient on examination of one proximal interphalangeal joint, the right wrist and the neck. Tenderness was also noted at the right knee and the right midfoot. All axial joints (neck, hips, and shoulders) were limited. Pressure on the sacroiliac joints was not painful. There was a pronounced swelling of the first metatarsophalangeal joint (MTP) on the right side, and of the second and third joints on the left side. The lumbar spine was painful and limited in motion. No enthesitis or tenosynovitis was noted.

Because of the strong suspicion of the presence of an inflammatory bowel disease, radiographic studies with barium were performed, but revealed no abnormalities.

Although specific criteria for the juvenile spondyloarthropathies $(\mathrm{SpA})$ are not available, the combination of teenage onset of arthritis of lower limb joints, limitation of the lower vertebral joints, morning stiffness, detection of the HLA-B27 antigen, and a brother with joint problems and uveitis in the presence of the HLA-B27 antigen made a diagnosis of juvenile $\mathrm{SpA}$ probable. Naproxen $15 \mathrm{mg} / \mathrm{kg} /$ day was started and within one week the joint complaints and the morning stiffness had improved significantly. A prolonged bleeding time (more than six minutes) became manifest, with nose bleeds and bloody stools. Haemoglobin decreased to $5 \cdot 1 \mathrm{mmol} / \mathrm{l}$. Medication was altered to tolmetine, which was less effective for the joint complaints. Because of the faecal blood loss, possible inflammatory changes in the gut were discussed again. Because of the capacity of non-steroidal anti-inflammatory drugs (NSAIDs) to inflame intestinal mucosa, these drugs were discontinued and oesophagoscopy
Table 1 Histology of biopsy specimens from the gastrointestinal tract

\begin{tabular}{|c|c|}
\hline Antrum & $\begin{array}{l}\text { Infiltrate between tubuli consisting of } \\
\text { lymphocytes, plasma cells and sporadic } \\
\text { granulocytes } \\
\text { Chronic active inflammation } \\
\text { Abundant presence of bacteria resembling } \\
\text { Helicobacter pylori }\end{array}$ \\
\hline Oesophagus & $\begin{array}{l}\text { Several dilated blood vessels } \\
\text { Low grade inflammatory infiltrate in stroma }\end{array}$ \\
\hline Duodenum & $\begin{array}{l}\text { Normal aspect of villi } \\
\text { Normal epithelial lining } \\
\text { Lymphocytic infiltration between tubuli }\end{array}$ \\
\hline $\begin{array}{l}\text { Ileocaecal area } \\
\text { Colon } \\
\text { Sigmoid }\end{array}$ & $\begin{array}{l}\text { Normal aspect of epithelial lining } \\
\text { Varying degree of subepithelial infiltrate of } \\
\text { lymphocytes and plasma cells } \\
\text { Low grade chronic inflammation }\end{array}$ \\
\hline
\end{tabular}

and colonoscopy with biopsy were performed. Histology of the biopsy specimens showed inflammation of oesophagus, duodenum and colon (table 1). Helicobacter pylori was universally present in the upper gastrointestinal tract. Enzymatic screening of the gut biopsy specimens confirmed a lactate deficiency.

\section{DISCUSSION}

\section{Differential diagnosis}

The child's initial presentation with intermittent arthralgia of the hip with limping could have reflected a variety of disorders (table 2 ).

Repeated episodes of coxalgia may be caused by recurrent transient synovitis, usually without systemic features. In other types of pauciarticular juvenile chronic arthritis (JCA), the hip joint is relatively spared. The differential diagnosis should also include osteoid osteoma. ${ }^{1}$ In most of these patients, nocturnal pain is more prominent, general features absent, and the effect of NSAIDs prompt. When a child has a painful joint, especially when associated with fever, the first diagnosis

Table 2 Differential diagnosis of hip pain in the older child and adolescent

\begin{tabular}{l} 
Inflammatory disorders \\
Irritable hip \\
Inflammatory synovitis \\
Forms of juvenile chronic arthritis \\
Spondyloarthropathies \\
Infectious \\
Septic arthritis \\
Osteomyelitis \\
Reactive arthritis \\
Mechanical causes \\
Legg-Calvé-Perthes disease \\
Epiphysiolysis \\
Slipped upper femoral epiphysis \\
Missed congenital dislocation of the hip \\
Osteoid osteoma \\
Avascular necrosis \\
Neoplastic diseases \\
Infiltrative marrow disorders \\
Leukaemia \\
Lymphoma \\
Neuroblastoma \\
Idiopathic/stress related disorders \\
Local abnormalities of the synovium \\
Generalised hypermobility \\
Idiopathic pain syndromes \\
Referred pain \\
Posttraumatic synovitis \\
Mixed rare causes \\
Bleeding diatheses \\
Epiphyseal dysplasias \\
Idiopathic chondrolysis \\
Gaucher's disease \\
Behçet's disease \\
Whipple's disease \\
SAPHO syndrome (synovitis, acne, pustulosis, hyperostosis, \\
osteitis) \\
Systemic lupus erythematosus \\
Sarcoidosis \\
Secondary osteoarthritis \\
\hline
\end{tabular}


to exclude is septic arthritis, as destruction of the joint is imminent.

Legg-Calvé-Perthes disease is usually a cause of limping in boys of a somewhat younger age than our patient.

Episodes of 'traumatic synovitis' in the hip caused by secondary osteoarthritis can occur in damaged hips, for example after septic arthritis, congenital dislocation of the hip, slipped capital femoral epiphysis, longstanding JCA, or in metabolic disorders such as hypothyroidism. The uneventful past history regarding the locomotor apparatus in our patient excluded these possibilities. Intermittent bleeding in a joint from a blood vessel malformation has been described. ${ }^{2}$ Synovial haemangiomas occur as part of a more generalised disorder, are rare in the hip and, moreover, are characterised by excruciating pain after mild traumas. Local abnormalities of the synovium of the hip, such as synovial sarcoma, may cause arthritis with intermittent limping, but synovial tumours usually present with painless extraarticular soft tissue swelling. Pigmented villonodular synovitis is also almost exclusively restricted to the knee joint, and was therefore an unlikely diagnosis in our patient.

Involvement of the gut was suspected when the boy returned with increasing anorexia, weight loss, and loose stools. In a child with chronic coxitis, inflammatory disorders of the bowel must be excluded even in the absence of intestinal complaints, especially when the haemoglobin concentration is low or growth is impaired. In coeliac disease, arthritis or arthralgia can be the first symptom, and its severity may vary with dietary measures. ${ }^{3}$ The absence of antigliadin and antireticulin antibodies made coeliac disease unlikely in our patient. The presence of oral ulcers and arthritis in combination with intestinal complaints could indicate Behçet's disease, systemic lupus erythematosus (SLE), or inflammatory bowel disease. On the basis of clinical and laboratory parameters these diagnoses could reasonably be excluded. Likewise, other diagnostic possibilities such as Whipple's disease and SAPHO syndrome (synovitis, acne, pustulosis, hyperostosis and osteitis) were highly unlikely. Inflammation of the gut caused by NSAIDs was unlikely as these drugs were discontinued several weeks before the biopsy specimens were obtained. ${ }^{4}$

The mouth ulcers, residua of skin lesions on the plantar surface of the feet, limitation of movement of the axial joints, and positive laboratory parameters for inflammation, in addition to the presence of the HLA-B27 antigen, led to the diagnosis of one of the juvenile forms of $\mathrm{SpA}$. Indirect support for this diagnosis was suggested by the improvement in morning stiffness after a hot shower and exercise, and the strong suspicion of $\mathrm{SpA}$ in an older brother. Inflammatory changes in the gut in patients with $\mathrm{SpA}$ have been described previously. ${ }^{5}$ There were no mucosal ulcers in our patient, and his biopsy specimens revealed no polymorphonuclear infiltration as described in ulcerative colitis; there was also no thickening of the bowel wall on the radiographic studies as would be seen in enteritis regionalis. The presence of Helicobacter pylori in the gastric mucosa and lactase deficiency are well known in the Moroccan population and probably not related to the joint disease in our patient.

\section{THE JUVENILE}

\section{SPONDYLOARTHROPATHIES}

Diagnostic criteria and epidemiology

Of the three major onset types of JCA, the pauci- or oligoarticular onset type is the most frequent $(60 \%)$. Pauciarticular disease may be subdivided into two subsets on clinical and immunogenetic grounds: early onset and late onset pauciarticular JCA, also known as the juvenile spondyloarthropathies. Some children cannot be included in either subgroup, while family studies suggest that the subtypes of pauciarticular JCA may be related. ${ }^{6}$ Diagnostic criteria for juvenile $\mathrm{SpA}$ are not yet available; those used in adults, such as the European Spondylarthropathy Study Group (ESSG) criteria, ${ }^{7}$ cannot be applied to children as signs and symptoms in children may differ significantly from those in adults. The ESSG and Amor criteria have been tested in a limited number of children, ${ }^{8}$ while the results of an international study are awaited.

In the meantime, juvenile $\mathrm{SpA}$ can be defined as a group of HLA-B27 related syndromes and clinical conditions affecting children and adolescents younger than 16 years who present with a broad spectrum of symptoms including arthritis, enthesitis, and tenosynovitis affecting peripheral joints and, variably, spine and sacroiliac joints in the course of their disease. ${ }^{9}$ Some patients present with seronegative enthesopathy and arthropathy syndrome which gradually evolves into ankylosing spondylitis (AS); other patients never develop this next stage.

In juvenile $\mathrm{SpA}$ there is a strong male preponderance of six to 10 boys to one girl. The age of onset is usually after the eighth birthday. Family histories are frequently positive for one of the forms of SpA.

With the recognition of formes frustes, the number of children suspected of having SpA and referred to a paediatric rheumatologist has increased. ${ }^{10}$ Diagnosis of undifferentiated forms of juvenile SpA may be difficult, as pain and swelling of posttraumatic or mechanical disorders may mimic the various clinical manifestations of SpA. Exact figures for the prevalence of the whole spectrum of juvenile SpA are not available.

\section{SUBGROUPS OF JUVENILE}

SPONDYLOARTHROPATHIES

Three evident subgroups of $\mathrm{SpA}$ can be recognised. The first subgroup and major disorder of this group is AS, another is formed by the reactive arthritides/Reiter's syndrome, and the third subgroup of the $\mathrm{SpA}$ is associated with codiseases such as inflammatory bowel diseases and psoriasis.

The clinical picture in a child with fully developed AS does not differ essentially from 
that in the adult with AS. It is estimated that approximately $10-15 \%$ of adult patients with AS develop symptomatic disease during childhood.

Reiter's syndrome refers to a clinical triad of sterile arthritis, conjunctivitis, and urethritis. Reactive arthritides follow an infection, in children usually of the gastrointestinal tract. Antibodies against various microbial antigens have been detected in the sera of these children: salmonella, shigella, yersinia, campylobacter, chlamydia and mycoplasma. ${ }^{9}$ The presence of the HLA-B27 antigen may even lead to $\mathrm{SpA}$ in patients with a reactive arthritis after a streptococcal infection. ${ }^{11}$ The list of microorganisms capable of triggering arthritis is still expanding. A defect in clearing certain infectious antigens is suspected.

In arthritis associated with inflammatory bowel diseases, reactive arthritis, and sometimes juvenile psoriatic arthritis, extra-articular symptoms may dominate the clinical picture at onset.

Arthritis associated with psoriasis is a heterogeneous group of disorders. The clinical picture of $\mathrm{SpA}$ with psoriasis is seen predominantly in patients with the HLA-B27 antigen. At onset joint involvement may be pauci- or polyarticular. ${ }^{12}$ There is currently discussion whether psoriatic arthritis exists as an entity, or whether the occurrence of psoriasis and arthritis is coincidental.

\section{Clinical features of the juvenile spondyloarthropathies}

ARTICULAR SYMPTOMS

The name juvenile SpA is misleading, as it suggests inflammation of the vertebral joints. Arthritis of axial joints or sacroiliitis may eventually develop in children with SpA, but asymmetrical peripheral arthritis in the lower extremities, enthesitis, and tenosynovitis are initially much more common. Arthritis of knee or ankle is a common presenting symptom in children with SpA. Less frequently, the small joints of the feet and hips are predominantly involved at onset. Children, unlike adults, do not usually present with inflammatory backpain. Prolonged (morning) stiffness is a common complaint.

The clinical course can be episodic, with flares following infection, or chronic. In severe current disease, destruction of the hip joints may eventually require arthroplasty..$^{13}$ Enthesitis is particularly noticeable at the calcanei, at the tibial and ischial tuberosities, and the iliac crest. $^{14}$ Tarsitis and inflammation of the tendon attachments around the heels may lead to considerable difficulty in walking. The synovial lining of the tendon sheath can be involved in the inflammatory process. This tenosynovitis can also lead to 'sausage' fingers and toes (dactylitis).

EXTRA-ARTICULAR MANIFESTATIONS

Juveniles with SpA may show considerable extra-articular symptoms, not only at onset but also during the course of their disease. These include malaise, weight loss, fever (even spiking), night sweats, uveitis, several skin rashes, and pyuria (Reiter's syndrome). Acute uveitis is the most frequent eye manifestation, but chronic blepharitis, conjunctivitis, or keratoconjunctivitis can also develop. Routine regular eye checks are advised only once a year, as the risk of developing chronic anterior uveitis is small. Valvular heart disease, particularly aortic insufficiency, has been described. ${ }^{15}$ In both differentiated and undifferentiated forms of $\mathrm{SpA}$, inflammation of the gastrointestinal tract resembling that of inflammatory bowel disease has been described in the absence of any bowel symptoms. ${ }^{5}$ Arthritides develop in about $15 \%$ of children with an inflammatory bowel disease. ${ }^{16}$ In the presence of the HLA-B27 antigen, the characteristic SpA axial pattern of joint involvement is seen. ${ }^{17}$

\section{Laboratory findings}

Laboratory investigation may show an increased ESR in about $75 \%$ of children with juvenile SpA. In some children, antinuclear antibodies can be detected in low titres with varying immunofluorescence patterns. Rheumatoid factors are negative. The HLA-B27 antigen is carried by $75-90 \%$ of the population, depending on the ethnic groups included.

\section{Radiographic features}

Children with SpA may show periarticular osteoporosis and signs of enthesitis at an early stage, and radiological abnormalities of the sacroiliac joints ${ }^{18}$ and lower lumbar spine in the long term, especially in the case of the low back or hip girdle symptoms. ${ }^{18} 19$ Periostitis can be demonstrated in children with dactylitis. Patients with heel pain may develop spurs at the attachment of the plantar fascia or Achilles tendon, or cortical bone erosions at or close to these sites.

\section{Treatment and outcome}

The treatment of a child with $\mathrm{SpA}$ is based upon intensive exercises, preferably in warm water. This regimen can be supported by the use of non-steroidal anti-inflammatory drugs. When arthritis is restricted to one or two joints, intra-articular injection with a long acting corticosteroid may be as helpful as in type 1 pauciarticular JCA. ${ }^{20}$ Disease modifying drugs in patients with persistent arthritis and enthesitis may be warranted. In the treatment of adults with $\mathrm{SpA}$, sulphasalazine is beneficial; ${ }^{21}$ controlled studies in juveniles are not yet available. Outcome depends on duration and severity of disease. Hip involvement appears to be associated with a poor functional outcome.

\section{Summary}

We have described a boy with a juvenile spondyloarthropathy who also showed inflammatory changes in the gut. Gastrointestinal 
symptoms initially indicated the presence of an inflammatory bowel disease, but this was excluded histopathologically.

\section{Key points}

- Spondyloarthropathies in children usually present as peripheral pauciarticular arthritis and not with inflammatory backpain as in adults.

- Malaise and anaemia with joint symptoms require exclusion of inflammatory bowel disease.

- The diagnostic criteria for spondyloarthropathies used in adults are not applicable to children.

1 Wakeley C J, Cobby M J. Teenage monoarthralgia. Ann Rheum Dis 1994; 53: 561-3.

2 Linsons M A. Synovial hemangioma as a cause of recurrent knee effusions. $\Im A M A$ 1979; 242: 2214-5.

3 Mäkki $M$, Hällstromm $\mathrm{O}$, Veronen $\mathrm{P}$, et al. Reticulin antibodies, arthritis and coeliac disease in children. Lancet 1988; 1: 479-80.

4 Hermaszewski R, Hayllar J, Woo P. Gastro-duodenal damage due to nonsteroidal anti-inflammatory drugs in children. Br $\mathcal{F}$ Rheumatol 1993; 32: 69-72.

5 Mielants H, Veys E M, Joos R, Cuvelier E, de Vos M, Proot F. Late onset pauciarticular juvenile chronic arthritis: relation to gut inflammation. $\mathcal{F}$ Rheumatol 1987; 14: 459-65.

6 Hertzberger-ten Cate R, Dijkmans B A C. Increased prevalence of spondylarthropathies in parents of patients with pauciarticular JCA, type 1. Clin Rheumatol 1993; 12: 361-5.
7 Dougados $M$, van der Linden $S$, Juhlin $R$, et al. The European Spondylarthropathy Study Group preliminary criteria for the classification of spondylarthropathy. Arthritis Rheum 1991; 34: 1218-27.

8 Prieur A M J, Listrat V, Dougados M, Amor B. Evaluation of the ESSG-criteria for juvenile spondylarthropathies: study of 310 consecutive children referred to one pediatric rheumatology center [abstract]. Arthritis Rheum 1990; 33 (suppl 9): D195.

9 Burgos-Vargas R. Spondylarthropathies and psoriatic arthritis in children. Curr Opin Rheumatol 1993; 5: arthritis

10 Rosenberg A M. Analysis of a pediatric rheumatology clinic population. F Rheumatol 1990; 17: 827-30.

11 Hubbard W N, Hughes G R V. Streptococci and reactive arthritis. Ann Rheum Dis 1982; 41: 435-7.

12 Southwood T R, Petty R E, Malleson P N, et al. Psoriatic arthritis in children. Arthritis Rheum 1989; 32: 1007-13.

13 Schaller J G. The seronegative sponylarthropathies of childhood. Clin Orthop 1979; 143: 76-83.

14 Rosenberg A M, Petty R E. The syndrome of seronegative enthesopathy and arthropathy in children. Arthritis Rheum 1984; 25: 1041-7.

15 Hull R G, Hall M A, Prasad A N. Aortic incompetence in pauciarticular juvenile chronic arthritis. Arch Dis Childh 1986; 61: 409-10.

16 Lindsley C, Schaller J G. Arthritis associated with inflammatory bowel disease in children. $\mathcal{F}$ Pediatr 1974; 84: 16-20.

17 Weiner S R, Clarke J, Taggart N, Utsinger P D. Rheumatic manifestations of inflammatory bowel disease. Semin Arthritis Rheum 1991; 20: 353-66.

18 Burgos-Vargas R, Petty R E. Juvenile ankylosing spondylitis. Rheum Dis Clin North Am 1992; 18: 123-42.

19 Jacobs J C, Berdon W E, Johnston A D. HLA-B27 associated spondyloarthritis and entheseopathy in childhood: ciated spondyloarthritis and entheseopathy in childhood: patients. ₹ Pediatr 1982; 100: 521-8.

20 Hertzberger-ten Cate $R$, de Vries-van der Vlugt B C M, van Suijlekom-Smit, Cats A. Intra-articular steroids in pauciarticular juvenile chronic arthritis, type 1 . Eur $\mathcal{f}$ Pediatr 1991; 150: 170-2.

21 Dougados M, van der Linden S J, Leirisalo-Repo M, et al. Sulfasalazine in spondylarthropathy: a randomized, multicentre, double-blind, placebo-controlled study. Arthritis Rheum 1995. In press. 